\title{
Riparian zones provide for distinct bird assemblages in forest mosaics of south-east Australia
}

\author{
Grant C. Palmer*, Andrew F. Bennett \\ Landscape Ecology Research Group, School of Life and Environmental Sciences, Deakin University, 221 Burwood Highway, \\ Burwood, Vic. 3125, Australia
}

\section{Introduction}

Riparian habitats are a distinctive component in many land scapes. Their topographic position, dendritic structure, high amount of edge area and connectivity through the landscape are characteristic features (Malanson, 1993; Forman, 1995). The value of riparian habitats for terrestrial wildlife has been investigated on a number of continents (Stauffer and Best, 1980; Decamps et al., 1987; Doyle, 1990; Warkentin et al.,
1995; Fisher and Goldney, 1997; Robertson et al., 1998), and fre quently they have been reported to harbour a rich and abun dant fauna in comparison with that of surrounding non riparian habitats (Thomas et al., 1978; Knopf and Samson, 1994; Lynch and Catterall, 1999; Woinarski et al., 2000). Further in heavily modified or cleared landscapes, riparian habitats of ten are prominent examples of the remaining natural or semi natural vegetation available to native biota (Gregory et al., 1991; Malanson, 1993; Lachavanne, 1997; Martin et al., 2006).

* Corresponding author: Present address: Centre for Environmental Management, School of Science and Engineering, University of Ballarat, P.O. Box 663, Ballarat, Vic. 3350, Australia. Tel.: +61 035327 9102; fax: +61 0353279240.

E mail address: g.palmer@ballarat.edu.au (G.C. Palmer). 
Consequently, throughout the world riparian zones are increasingly being promoted as key areas for biodiversity con servation (Knopf et al., 1988; Catterall, 1993; Malanson, 1993).

The value of riparian zones for birds has been well demon strated in semi arid and arid landscapes (Anderson and Ohm art, 1977; Shurcliff, 1980; Szaro, 1980; Knopf, 1985; Szaro and Jakle, 1985; Fleishman et al., 2002). In these situations, condi tions in the riparian zone (e.g. moisture regimes, nutrient availability) often contrast strongly with those predominating in the surrounding non riparian matrix (Gregory et al., 1991; Malanson, 1993). This leads to distinct patterning of vegeta tion associations in the landscape (Austin et al., 1996) and birds respond positively to such diversity of habitats (Cody, 1993; Borchert, 2003). Riparian habitats in managed land scapes, such as remnant vegetation along streams in agricul tural areas (Crome et al., 1995; Fisher and Goldney, 1997; Kilgo et al., 1998; Jansen and Robertson, 2001; Martin et al., 2006) and among plantation forests (Friend, 1982; Armstrong and van Hensbergen, 1994; Hodges and Krementz, 1996; Linden mayer et al., 2002; Conner et al., 2004) have also been a focus for research effort and are considered important for avifaunal conservation. In these environments too, there is a marked contrast between the vegetation of the riparian zone and that of adjacent land.

Less attention has been given to the role of riparian habi tats in largely intact landscapes, where riparian and adjacent non riparian habitats maintain continuous vegetation cover (Catterall, 1993; Murray and Stauffer, 1995; Woinarski et al., 2000). In continuous forests in mesic environments, for exam ple, there may be less contrast between riparian zones and adjacent vegetation as habitat for birds, due to the greater availability of moisture across the landscape and the continu ity of forest cover. Some studies in such environments have found bird assemblages in non riparian habitats to have equal or greater species richness and diversity than nearby riparian assemblages (McGarigal and McComb, 1992; Pearson and Manuwal, 2001; Shirley and Smith, 2005).

In this study, the use of riparian zones by birds in contin uous forest landscapes in mesic southeastern Australia was investigated. The study was based on explicit contrasts of the avifauna and habitat characteristics at 30 pairs of riparian and adjacent non riparian sites in extensive foothill forests in the Victorian Highlands. There were three main objectives:

1. To compare structural and floristic features of riparian and non riparian vegetation to identify attributes that may contribute to distinctive habitats for birds.

2. To quantify the bird assemblages of riparian and non riparian habitats to investigate any differences in species richness and abundance between habitat types.

3. To compare the composition of avifaunal assemblages between riparian and non riparian habitats to identify the strength of species' relationships with the riparian zone.

\section{Methods}

\subsection{Study area}

The study was conducted in the Victorian Highlands, south east Australia. Three extensive forest areas were investigated:
Bunyip State Park $\left(37^{\circ} 56^{\prime} \mathrm{S}, 145^{\circ} 35^{\prime} \mathrm{E}\right)$, Kinglake National Park $\left(37^{\circ} 29^{\prime} \mathrm{S}, 145^{\circ} 22^{\prime} \mathrm{E}\right)$ and Marysville State Forest $\left(37^{\circ} 34^{\prime} \mathrm{S}\right.$, $145^{\circ} 41^{\prime} \mathrm{E}$ ). Mean annual rainfall in the study area is 900 $1400 \mathrm{~mm}$, with most rain falling between April and Septem ber. The area experiences dry, hot summers $\left(25^{\circ} \mathrm{C}\right.$ January average daily maxima) and cool, damp winters $\left(12^{\circ} \mathrm{C}\right.$ July average daily maxima).

Riparian zones are interspersed in the mixed Eucalyptus forest mosaic as relatively narrow bands of vegetation along the dendritic stream network that drains both the coastal and inland fall of the Great Dividing Range in this region. The streamside vegetation is typically classified (by the Department of Sustainability and Environment, Victoria) as Riparian Forest ecological vegetation class (EVC). A wide range of other vegetation associations occur in upland areas of the landscape. On protected south facing slopes there are tall, moist forest associations (Wet Forest, Damp Forest and Shrubby Foothill Forest ecological vegetation classes) (Com monwealth and Victorian Regional Forest Agreement Steering Committee, 1997). Low, heathy forests and woodlands domi nate on the drier, gently sloping north facing aspects, and characteristically support a dense shrub layer. Tall open for ests with a grass, herb and shrub understorey occur on inter mediate slopes. Notably, throughout the landscape a continuous eucalypt tree canopy is maintained along the gra dient from riparian to upland habitats.

\subsection{Study sites}

Site selection was driven by the availability of extensive ripar ian zones located in forested catchments that displayed no evidence of recent disturbance. Potential sites were identified from vegetation maps (ecological vegetation classes) of the re gion. Stretches of continuous Riparian Forest that fringed perennial mid order stream systems (stream order 3 to 5 , stream width $18 \mathrm{~m}$ ) and were greater than $5000 \mathrm{~m}$ in length were sought. Of potential stream systems, six were selected and a total of 30 sites was located as follows: Black Snake Creek (n 10 sites), Bunyip River (4), Diamond Creek (6) (all in Bunyip State Park), Island Creek (4), Captain Creek (1) (both Kinglake National Park) and Acheron River (5) (Marysville State Forest). Riparian sites were positioned alongside the stream, with the site boundary within $10 \mathrm{~m}$ of the stream edge.

Non riparian sites were positioned parallel to their ripar ian partner on a facing slope at a distance of approximately $750 \mathrm{~m}$. Non riparian sites represent a range of ecological veg etation classes; Wet Forest ( $n \quad 1$ in Bunyip State Park), Damp Forest (4 Bunyip State Park and Marysville State Forest), Shrubby Foothill Forest (4 Bunyip State Park), Herb rich Foot hill Forest (4 Marysville State Forest), Lowland Forest (6 Bun yip State Park and Kinglake National Park), Heathy Dry Forest (3 Kinglake National Park) and Heathy Woodland (8 Bunyip State Park). A distance of at least $1000 \mathrm{~m}$ was main tained between site pairs.

\subsection{Habitat characteristics}

Data on habitat structure and floristic composition were gath ered at all sites (Table 1). Habitat structure assessments were based on vegetation life forms. All trees were identified to 


$\begin{aligned} & \text { Table 1 - Description of habitat variables measured at } \\ & \text { riparian and non-riparian sites in the Victorian } \\ & \text { Highlands }\end{aligned}$
Variable

species level, counted and determined to be either canopy forms or mid storey forms, within a 0.25 ha quadrat $(100 \mathrm{~m} \times$ $25 \mathrm{~m}$ ) at each site. The diameter at breast height (dbh) of each tree was measured and assigned to one of six size classes ( $\leqslant 10 \mathrm{~cm} \mathrm{dbh,} 1120 \mathrm{~cm}, 2140 \mathrm{~cm}, 4160 \mathrm{~cm}, 6180, \geqslant 81 \mathrm{~cm}$ ). The cover (\%) of the canopy and mid storey tree layers was visually estimated. Dead standing trees were similarly mea sured and counted, and categorised into two size classes ( $\leqslant 10 \mathrm{~cm} \mathrm{dbh},>10 \mathrm{~cm}$ ). Trees bearing mistletoe (Amyema spp.) or with hollows visible from the ground were tallied. For shrub assessments, a randomly placed $25 \mathrm{~m} \times 25 \mathrm{~m}$ quadrat was used. Shrubs were identified, counted and assigned to one of three height classes $(<1,12,>2 \mathrm{~m})$. The cover $(\%)$ of each shrub species was also recorded in each height class. The cover (\%) of a suite of vegetation life forms (e.g. tree ferns, low ferns, grasses, sedges) was also visually estimated in $10 \%$ intervals within this quadrat (Table 1). Cover of bare ground, fine litter and ground vegetation was assessed in four $25 \mathrm{~m}^{2}(5 \mathrm{~m} \times 5 \mathrm{~m})$ quadrats and average values generated for each site. The extent of coarse woody debris in two size categories ( $\leqslant 50 \mathrm{~cm}$ diameter, $>50 \mathrm{~cm}$ diameter) was measured as the number of intercepts along a $100 \mathrm{~m}$ transect centrally positioned at each site.

\subsection{Bird survey}

Bird assemblages were sampled using a fixed point count method (Pyke and Recher, 1984). Fixed points were centrally located $50 \mathrm{~m}$ apart in two adjoining plots, each $50 \mathrm{~m} \times 50 \mathrm{~m}$, yielding a combined sampling area of 0.5 ha at a site. At each fixed point the survey time was standardised to $8 \mathrm{~min}$. Upon completion of the survey at the first point, the observer (GP) moved to the next point and commenced another $8 \mathrm{~min}$ count, a standard 2 min after completion of the first. All birds seen or heard within the two plots were recorded. Occurrence of birds within plots and movements between plots were clo sely monitored to avoid duplication of individual observations wherever possible. The data reported here were pooled from both plots at each site. The taxonomy for bird species follows Christidis and Boles (1994).

During the study, each site was visited on 29 occasions, a total of 3480 point counts across the 60 sites. Each site was surveyed five times per season (winter, spring, summer and autumn) between July 2001 and December 2002. Surveys were conducted throughout the day in suitable still and dry condi tions. Nocturnal surveys were not undertaken and therefore species active at night (e.g. owls and nightjars) were poorly sampled. Due to the constraints posed by geographic separa tion, sites were grouped by stream units and the order of site pair surveys was randomised within these units.

\subsection{Analysis}

Differences between the habitat structure of riparian and non riparian habitats were tested by using analysis of similar ity (ANOSIM) in the PRIMER software package (Clarke and Gor ley, 2001). For all analyses, a significance level of $p \quad 0.05$ was employed. A related procedure, similarity percentage (SIM PER), was then used to identify the physiognomic variables that contribute most to the similarities within site groups (i.e. riparian, non riparian) and to the dissimilarities between groups based on contributions of variables to the Bray Curtis similarity matrix (PRIMER software package) (Clarke and Gor ley, 2001). Habitat variables were standardised for analyses because they were measured on different scales.

To investigate floristic associations of sites, a modified 'importance value' (Mueller Dombois and Ellenberg, 1974) was employed. For tree species, this index was calculated by summing the proportional contribution of each species at a site to the total basal area (relative dominance) and total stem density (relative density). For shrubs, the index generated for each species at a site was the sum of the percentage of total shrub cover and percentage of total number of shrubs. Impor tance indices, therefore, have values from 0 to 200 for identi fied plant species at a given site. Importance values for tree and shrub species at each site were tabulated and converted to a similarity by site matrix using the Bray Curtis similarity measure. The ANOSIM and SIMPER procedures were then used for comparisons between riparian and non riparian sites, using square root transformed variables to reduce the influence of abundant species.

Bird species observations were compiled and pooled for all 29 visits to each site. Species richness values were analysed by using a paired t test to compare between riparian and non riparian sites for each pair. Species abundance and species diversity (Shannon Weiner diversity index) values were also analysed using paired $t$ tests. ANOSIM and SIMPER procedures (Clarke and Gorley, 2001) were used to test for differences in 
species composition between riparian and non riparian sites and to identify species contributing most to the similarity within site types (riparian or non riparian) and the dissimilar ity between site types (riparian v non riparian). Again, variables were square root transformed to reduce the influence of abundant species and give greater weight to less common species.

An ordination of bird assemblages at each site was con structed by using multidimensional scaling (MDS), based on a Bray Curtis similarity matrix. To assist in interpreting the ordination, Spearman rank correlations were calculated be tween the ordination dimensions and all measured physiog nomic and floristic variables for each site. This enabled the variables most strongly correlated with each of the MDS dimensions to be identified.

\section{Results}

\subsection{Habitat characteristics}

Habitat structure differed significantly (ANOSIM, R 0.656, $p<0.001$ ) between riparian and non riparian sites. The most distinctive features of riparian habitats were the taller canopy height, a ground layer with extensive cover of fine litter and ground vegetation, large amounts of coarse woody debris ( $\leqslant 50 \mathrm{~cm}$ diameter) and a dense cover of mid storey trees (Ta ble 2). The characteristic features of non riparian habitats in cluded a relatively dense canopy cover, a ground layer dominated by ground vegetation and fine litter, high cover of grasses and a high density of canopy forming trees in the smaller size classes (Table 2).

Table 2 - Habitat variables characteristic of riparian and non-riparian habitats in the Victorian Highlands

\begin{tabular}{|c|c|c|c|c|c|}
\hline \multirow[t]{2}{*}{ Variable } & \multicolumn{2}{|c|}{ Similarity } & \multirow{2}{*}{$\frac{\text { Dissimilarity }}{\text { RIP v NR }}$} & \multicolumn{2}{|c|}{ Variable means } \\
\hline & RIP & NR & & RIP & NR \\
\hline \multicolumn{6}{|l|}{ Structural variables } \\
\hline Canopy height & 8.0 & 6.2 & & 39.5 & 30.6 \\
\hline Fine litter & 7.6 & 6.6 & 3.5 & 44.7 & 44.5 \\
\hline Coarse woody debris $(\leqslant 50 \mathrm{~cm})$ & 7.2 & 5.2 & & 140.0 & 103.6 \\
\hline Ground vegetation & 7.1 & 6.7 & 4.1 & 43.8 & 51.7 \\
\hline Mid storey trees & 6.7 & & 8.5 & 37.0 & 3.0 \\
\hline Sedges & 6.2 & & 5.5 & 39.0 & 12.2 \\
\hline Ground ferns & 5.6 & & 5.7 & 35.3 & 17.8 \\
\hline Tall shrubs & 4.9 & & & 25.7 & 23.0 \\
\hline Tree ferns & 4.0 & & 5.2 & 16.0 & 2.3 \\
\hline Bare ground & 3.8 & & & 13.5 & 9.7 \\
\hline Grasses & 3.0 & 6.3 & 5.6 & 14.7 & 50.0 \\
\hline Canopy cover & & 8.7 & & 35.5 & 56.0 \\
\hline Tree density (21 $40 \mathrm{~cm}$ dbh) & & 6.7 & 4.9 & 44.8 & 164.0 \\
\hline Shrub cover $(\leqslant 1 \mathrm{~m})$ & & 4.5 & 4.5 & 4.2 & 22.8 \\
\hline Dead standing trees $(\leqslant 10 \mathrm{~cm} \mathrm{dbh})$ & & 4.3 & 3.2 & 38.8 & 95.6 \\
\hline Shrub cover $(\geqslant 2 \mathrm{~m})$ & & 4.2 & 3.5 & 25.7 & 23.0 \\
\hline Tree density ( $\leqslant 10 \mathrm{~cm} \mathrm{dbh)}$ & & 5.1 & 7.3 & 7.6 & 137.6 \\
\hline Tree density (11 $20 \mathrm{~cm} \mathrm{dbh}$ ) & & 5.0 & 5.7 & 12.0 & 118.4 \\
\hline Shrub cover (1 $2 \mathrm{~m})$ & & 4.9 & 3.6 & 9.7 & 25.0 \\
\hline \multicolumn{6}{|c|}{ Tree and shrub species (Importance Values) } \\
\hline Comprosma quadrifida & 31.4 & & 9.4 & 120.9 & 9.2 \\
\hline Pomaderris aspera & 16.3 & & 5.7 & 41.0 & 0.8 \\
\hline Acacia melanoxylon & 9.3 & & 3.5 & 16.4 & 0.1 \\
\hline A. dealbata & 7.9 & & 3.0 & 12.3 & 0.8 \\
\hline Eucalyptus viminalis & 6.5 & & 3.7 & 25.3 & \\
\hline E. radiata & & 23.33 & 5.4 & 16.8 & 66.3 \\
\hline E. obliqua & & 13.34 & 4.5 & 16.8 & 48.1 \\
\hline Hakea sericea & & 8.1 & 3.3 & 14.0 & 10.4 \\
\hline Banksia spinulosa & & 6.9 & 2.9 & 0.8 & 15.9 \\
\hline E. sieberi & & 6.8 & 3.5 & & 29.0 \\
\hline E. baxteri & & 5.5 & 3.2 & 1.0 & 25.4 \\
\hline Lomatia ilicifolia & & 4.1 & 1.9 & & 7.8 \\
\hline Leptospermum continentale & & 3.9 & 2.8 & & 19. 9 \\
\hline Melaleuca squarrosa & & & 3.1 & 22.8 & \\
\hline E. camphora & & & 2.5 & 23.2 & \\
\hline Spyridium parvifolium & & & 2.3 & & 19.1 \\
\hline Platylobium formosum & & & 2.2 & 6.0 & 11.1 \\
\hline Epacris impressa & & & 1.9 & 0.6 & 7.6 \\
\hline L. lanigerum & & & 1.4 & 14.9 & \\
\hline
\end{tabular}

Values represent the percentage contributions to similarity within riparian (RIP) and non riparian (NR) sites, and dissimilarities between riparian and non riparian sites (RIP vs. NR) based on Bray Curtis indices (SIMPER). Analyses were conducted separately for structural variables and floristic composition of trees and shrubs. 
Variables that contributed to the similarities within ripar ian and non riparian habitats also contributed to the dissim ilarities between these habitat types (Table 2). In particular, contrasts between habitat types were derived from dissimilar ities in the structure of the tree layers. Riparian habitats were near exclusive in containing a mid storey tree layer domi nated by species such as scented paperbark (Melaleuca squar rosa), hazel pomaderris (Pomaderris aspera), blackwood (Acacia melanoxylon) and silver wattle (A. dealbata) (Table 2). The distribution of tree size classes also contributed strongly to dissimilarities, with the density of canopy trees in the size classes $\leqslant 10 \mathrm{~cm}$ dbh, $1120 \mathrm{~cm}$ dbh and $2140 \mathrm{~cm}$ dbh being al most twenty, nine and three times, respectively, greater in non riparian habitats (Table 2). Other variables that contrib uted to the dissimilarities between habitat types included cover of ground ferns (twice as great in riparian habitats) and cover of sedges (three times greater in riparian habitats) (Table 2).

Differences in the floristic composition of riparian and non riparian habitats were highly significant (ANOSIM, $R$ $0.814, p<0.001$ ). Five species of trees and shrubs contrib uted approximately $70 \%$ of the similarity within riparian habitats (Table 2). None of these were included in the eight species contributing to $70 \%$ of the similarity in non riparian habitats (Table 2). Dissimilarity between riparian and non riparian sites was generated either by the unique occur rence of tree and shrub species in one habitat type or from large differences in importance values of species between types (Table 2).

\subsection{Bird assemblages}

Eighty eight bird species were recorded at sites during sur veys (Table 3). The brown thornbill and striated thornbill (see Table 3 for scientific names) were recorded at all riparian and non riparian sites. Other species recorded at $>90 \%$ of sites included grey fantail, spotted pardalote, yellow faced honeyeater, crimson rosella, golden whistler, eastern spine bill, grey shrike thrush, white throated treecreeper and red wattlebird (Table 3). Fifteen species were recorded only at riparian sites; of these, Australian shelduck, sulphur crested cockatoo, yellow tufted honeyeater, pink robin, satin bower bird, red browed finch and swamp harrier were recorded at more than one site (Table 3). Of ten species recorded exclu sively at non riparian sites, only wedge tailed eagle, buff rumped thornbill and yellow thornbill were recorded at multi ple sites (Table 3).

Bird assemblages of riparian zones were significantly ri cher in species compared with non riparian habitats (paired $t$ test, $t \quad 10.16$, df $29, p<0.001$ ). The mean species richness of assemblages in riparian habitats was 36.9 species $( \pm 4.94$ SD, range 28 46), compared with 25.5 ( \pm 3.92 SD, range 18 33) for non riparian habitats. In all cases, riparian sites supported higher species richness than occurred at their non riparian site partner.

The relative abundance of birds recorded in riparian habi tats, 35.5 individuals ha ${ }^{1}( \pm 8.12 \mathrm{SD}$, range 21.350 .3$)$, was also significantly greater ( $t \quad 12.17, \mathrm{df} \quad 29, p<0.001)$, than that reg istered in non riparian habitats, 14.0 individuals ha ${ }^{1}( \pm 4.95$ SD, range 5.7 23.5). The diversity of bird assemblages was also significantly greater ( $t$ 2.93, df 29, $p$ 0.003) in riparian habitats $\left(H^{\prime} 3.09, S D \pm 0.23\right)$ compared with that in non riparian habitats $\left(H^{\prime} \quad 2.28, S D \pm 0.31\right)$.

The composition of bird assemblages differed significantly between riparian and adjacent non riparian habitats (ANO SIM, $R \quad 0.713, p<0.001$ ) (Fig. 1). A SIMPER analysis showed that for riparian sites, 13 bird species contributed approxi mately $70 \%$ of the similarity among assemblages occurring at these sites. Those contributing most to the similarity of riparian assemblages included brown thornbill (10.9\%), stri ated thornbill $(8.9 \%)$, white browed scrubwren $(7.5 \%)$, yel low faced honeyeater (6.0\%) and grey fantail (5.9\%). Eleven species contributed to $70 \%$ of the similarity among assem blages at non riparian sites. The greatest contributors were striated thornbill (15.8\%), brown thornbill (12.8\%), red wattle bird (6.3\%), grey fantail (6.2\%) and eastern spinebill (5.4\%). Half (7 of 14 species) of the species contributing most to the similarities within riparian or non riparian habitats were common to both: brown and striated thornbills, yellow faced honeyeater, grey fantail, spotted pardalote, golden whistler and white throated treecreeper.

Twenty seven species accounted for $70 \%$ of the dissimilar ity between bird assemblages of riparian and non riparian habitats. The greatest contributors were white naped honey eater $(6.7 \%)$, white browed scrubwren (5.7\%), brown thornbill (4.3\%), yellow faced honeyeater (4.1\%) and silvereye (3.9\%). By comparing the mean abundance of birds in each habitat type (Table 3), it is evident that species contributions to dissimilar ities were predominantly generated by those with large con trasts in relative abundance between habitat types. Species more abundant in riparian habitats included white naped honeyeater, brown thornbill, white browed scrubwren, silver eye and yellow faced honeyeater (Table 3). Overall 36\% (n 32) of species attained a greater abundance in riparian habitats. Those with higher abundance in non riparian habitats, and contributing strongly to dissimilarities between habitat types, included red wattlebird (2.6\%), superb fairy wren $(2.0 \%)$ and rufous whistler (1.4\%) (Table 3).

An MDS ordination of sites based on the composition of their bird assemblages clearly displayed the contrast between riparian and non riparian sites (Fig. 1) and provided a good fit to the data (stress 0.1 ) (Clarke and Gorley, 2001). There was a distinguishable clustering of sites, based on bird species com position, which corresponded with ecological vegetation clas ses (Fig. 1). Riparian sites (i.e. Riparian Forest) were strongly correlated at the positive end of MDS dimension 1 (MDS1) (Fig. 1). There was greater variation among non riparian sites in the composition of bird assemblages, with sites spread in ordination space in a pattern reflecting their vegetation type (Fig. 1).

Correlation analyses (Spearman rank correlation) showed that many habitat variables were significantly correlated with MDS1. This ordination dimension generally represents a gra dient from wet to drier forest types. Variables positively corre lated with MDS1 were characteristic of riparian habitats (Fig. 1), including foliage cover of mid storey trees $\left(r_{\mathrm{s}} \quad 0.825\right.$, $p<0.01)$, cover of tree ferns $\left(r_{\mathrm{s}} \quad 0.750, p<0.01\right)$, ground ferns $\left(r_{\mathrm{s}} 0.438, p<0.01\right)$, creepers $\left(r_{\mathrm{s}} 0.485, p<0.01\right)$, sedges $\left(r_{\mathrm{s}} \quad 0.409, p<0.01\right)$ and canopy height $\left(r_{\mathrm{s}} \quad 0.446, p<0.01\right)$. Variables negatively correlated with MDS1 were indicative of 
Table 3 - The relative abundance of bird species (individuals $\mathrm{ha}^{-1}$ ) recorded during point counts at riparian and non-riparian sites in the Victorian Highlands

\begin{tabular}{|c|c|c|c|c|c|c|c|}
\hline \multirow[t]{2}{*}{ Common name } & \multirow[t]{2}{*}{ Scientific name } & \multicolumn{3}{|c|}{ Riparian } & \multicolumn{3}{|c|}{ Non riparian } \\
\hline & & Sites & Mean & $\mathrm{SE}$ & Sites & Mean & $\mathrm{SE}$ \\
\hline Australian shelduck & Tadorna tadornoides & 2 & 0.01 & 0.01 & & & \\
\hline Australian wood duck & Chenonetta jubata & 1 & $<0.01$ & $<0.01$ & & & \\
\hline Pacific black duck & Anas superciliosa & 1 & 0.01 & 0.01 & & & \\
\hline Whistling kite & Haliastur sphenurus & 1 & $<0.01$ & $<0.01$ & & & \\
\hline Brown goshawk & Accipiter fasciatus & 6 & 0.02 & 0.01 & 2 & $<0.01$ & $<0.01$ \\
\hline Collared sparrowhawk & A. cirrhocephalus & 1 & $<0.01$ & $<0.01$ & 1 & $<0.01$ & $<0.01$ \\
\hline Wedge tailed eagle & Aquila audax & & & & 2 & 0.01 & 0.01 \\
\hline Little eagle & Hieraaetus morphnoides & 1 & $<0.01$ & $<0.01$ & 2 & 0.01 & 0.01 \\
\hline Swamp harrier & Circus approximans & 2 & $<0.01$ & $<0.01$ & & 0.00 & 0.00 \\
\hline Peregrine falcon & Falco peregrinus & & & & 1 & $<0.01$ & $<0.01$ \\
\hline Brush bronzewing & Phaps elegans & & & & 1 & $<0.01$ & $<0.01$ \\
\hline Yellow tailed black cockatoo & Calyptorhynchus funereus & 8 & 0.08 & 0.03 & 8 & 0.06 & 0.02 \\
\hline Gang gang cockatoo & Callocephalon fimbriatum & 14 & 0.10 & 0.02 & 8 & 0.07 & 0.03 \\
\hline Sulphur crested cockatoo & Cacatua galerita & 8 & 0.08 & 0.03 & & & \\
\hline Musk lorikeet & Glossopsitta concinna & 2 & $<0.01$ & $<0.01$ & 1 & $<0.01$ & $<0.01$ \\
\hline Little lorikeet & G. pusilla & 2 & 0.01 & 0.01 & 1 & $<0.01$ & $<0.01$ \\
\hline Purple crowned lorikeet & G. porphyrocephala & 1 & $<0.01$ & $<0.01$ & & & \\
\hline Australian king parrot & Alisterus scapularis & 12 & 0.06 & 0.02 & 10 & 0.04 & 0.01 \\
\hline Crimson rosella & Platycercus elegans & 30 & 0.51 & 0.07 & 28 & 0.47 & 0.11 \\
\hline Eastern rosella & P. eximius & 8 & 0.03 & 0.01 & 9 & 0.07 & 0.02 \\
\hline Swift parrot & Lathamus discolor & 1 & $<0.01$ & $<0.01$ & & & \\
\hline Pallid cuckoo & Cuculus pallidus & 1 & $<0.01$ & $<0.01$ & 1 & $<0.01$ & $<0.01$ \\
\hline Fan tailed cuckoo & Cacomantis flabelliformis & 13 & 0.04 & 0.01 & 11 & 0.04 & 0.01 \\
\hline Shining bronze cuckoo & Chrysococcyx lucidus & 20 & 0.07 & 0.01 & 10 & 0.03 & 0.01 \\
\hline Southern boobook & Ninox novaeseelandiae & 1 & $<0.01$ & $<0.01$ & & & \\
\hline Australian owlet nightjar & Aegotheles cristatus & & & & 1 & $<0.01$ & $<0.01$ \\
\hline White throated needletail & Hirundapus caudacutus & 1 & $<0.01$ & $<0.01$ & 5 & 0.06 & 0.03 \\
\hline Laughing kookaburra & Dacelo novaeguineae & 19 & 0.11 & 0.03 & 15 & 0.08 & 0.02 \\
\hline Sacred kingfisher & Todiramphus sanctus & 10 & 0.04 & 0.01 & 1 & $<0.01$ & $<0.01$ \\
\hline Superb lyrebird & Menura novaehollandiae & 15 & 0.08 & 0.02 & 6 & 0.03 & 0.02 \\
\hline White throated treecreeper & Cormobates leucophaeus & 30 & 0.54 & 0.05 & 26 & 0.43 & 0.06 \\
\hline Red browed treecreeper & Climacteris erythrops & 27 & 0.50 & 0.09 & 9 & 0.08 & 0.03 \\
\hline Superb fairy wren & Malurus cyaneus & 23 & 0.40 & 0.07 & 23 & 0.46 & 0.09 \\
\hline Southern emu wren & Stipituris malachurus & 4 & 0.03 & 0.02 & 15 & 0.24 & 0.07 \\
\hline Spotted pardalote & Pardalotus punctatus & 30 & 0.80 & 0.07 & 29 & 0.40 & 0.05 \\
\hline Striated pardalote & P. striatus & 30 & 1.31 & 0.15 & 23 & 0.17 & 0.03 \\
\hline White browed scrubwren & Sericornis frontalis & 30 & 2.75 & 0.12 & 17 & 0.37 & 0.10 \\
\hline Large billed scrubwren & S. magnirostris & 22 & 0.23 & 0.05 & 1 & $<0.01$ & $<0.01$ \\
\hline White throated gerygone & Gerygone olivacea & 1 & $<0.01$ & $<0.01$ & & & \\
\hline Brown thornbill & Acanthiza pusilla & 30 & 5.61 & 0.21 & 30 & 2.29 & 0.25 \\
\hline Buff rumped thornbill & A. reguloides & & & & 4 & 0.09 & 0.05 \\
\hline Yellow thornbill & A. nana & & & & 5 & 0.03 & 0.02 \\
\hline Striated thornbill & A. lineata & 30 & 4.52 & 0.37 & 30 & 3.16 & 0.31 \\
\hline Red wattlebird & Anthochaera carunculata & 28 & 0.54 & 0.10 & 27 & 1.21 & 0.27 \\
\hline Noisy miner & Manorina melanocephala & & & & 1 & $<0.01$ & $<0.01$ \\
\hline Lewin's honeyeater & Meliphaga lewinii & 15 & 0.15 & 0.03 & 1 & $<0.01$ & $<0.01$ \\
\hline Yellow faced honeyeater & Lichenostomus chrysops & 30 & 2.03 & 0.15 & 29 & 0.28 & 0.05 \\
\hline White eared honeyeater & L. leucotis & 22 & 0.17 & 0.03 & 7 & 0.03 & 0.02 \\
\hline Yellow tufted honeyeater & L. melanops & 5 & 0.30 & 0.16 & & & \\
\hline Brown headed honeyeater & Melithreptus brevirostris & 21 & 0.22 & 0.06 & 12 & 0.09 & 0.04 \\
\hline White naped honeyeater & M. lunatus & 29 & 4.33 & 0.74 & 15 & 0.15 & 0.05 \\
\hline Crescent honeyeater & Phylidonyris pyrrhoptera & 28 & 0.60 & 0.09 & 15 & 0.17 & 0.05 \\
\hline New Holland honeyeater & P. novaehollandiae & 7 & 0.09 & 0.06 & 1 & $<0.01$ & $<0.01$ \\
\hline Eastern spinebill & Acanthorhynchus tenuirostris & 28 & 0.51 & 0.07 & 29 & 0.63 & 0.10 \\
\hline Scarlet robin & Petroica multicolor & 2 & 0.01 & 0.01 & 19 & 0.16 & 0.04 \\
\hline Flame robin & P. phoenicea & & & & 3 & 0.01 & 0.01 \\
\hline Rose robin & P. rosea & 30 & 0.40 & 0.03 & 5 & 0.03 & 0.02 \\
\hline Pink robin & P. rodinogaster & 6 & 0.02 & 0.01 & & & \\
\hline Eastern yellow robin & Eopsaltria australis & 30 & 0.98 & 0.09 & 21 & 0.25 & 0.05 \\
\hline Eastern whipbird & Psophodes olivaceus & 19 & 0.19 & 0.04 & 1 & $<0.01$ & $<0.01$ \\
\hline Varied sittella & Daphoenositta chrysoptera & 21 & 0.21 & 0.04 & 17 & $\begin{array}{c}0.18 \\
\text { (continued }\end{array}$ & $\begin{array}{c}0.04 \\
\text { next pa }\end{array}$ \\
\hline
\end{tabular}


Table 3 - (continued)

\begin{tabular}{|c|c|c|c|c|c|c|c|}
\hline \multirow[t]{2}{*}{ Common name } & \multirow[t]{2}{*}{ Scientific name } & \multicolumn{3}{|c|}{ Riparian } & \multicolumn{3}{|c|}{ Non riparian } \\
\hline & & Sites & Mean & $\mathrm{SE}$ & Sites & Mean & $\mathrm{SE}$ \\
\hline Crested shrike tit & Falcunculus frontatus & 16 & 0.08 & 0.02 & 1 & $<0.01$ & $<0.01$ \\
\hline Olive whistler & Pachycephala olivacea & 21 & 0.11 & 0.02 & 3 & 0.01 & 0.00 \\
\hline Golden whistler & P. pectoralis & 30 & 0.90 & 0.08 & 28 & 0.20 & 0.03 \\
\hline Rufous whistler & P. rufiventris & 15 & 0.08 & 0.02 & 26 & 0.24 & 0.04 \\
\hline Grey shrike thrush & Colluricincla harmonica & 28 & 0.21 & 0.03 & 28 & 0.27 & 0.03 \\
\hline Leaden flycatcher & Myiagra rubecula & & & & 1 & $<0.01$ & $<0.01$ \\
\hline Satin flycatcher & M. cyanoleuca & 22 & 0.19 & 0.04 & 6 & 0.03 & 0.01 \\
\hline Rufous fantail & Rhipidura rufifrons & 28 & 0.37 & 0.04 & 5 & 0.02 & 0.01 \\
\hline Grey fantail & R. fuliginosa & 30 & 1.84 & 0.11 & 29 & 0.63 & 0.08 \\
\hline Black faced cuckoo shrike & Coracina novaehollandiae & 11 & 0.04 & 0.01 & 13 & 0.08 & 0.02 \\
\hline Olive backed oriole & Oriolus sagittatus & 4 & 0.02 & 0.01 & 13 & 0.06 & 0.01 \\
\hline Dusky woodswallow & Artamus cyanopterus & 2 & 0.09 & 0.07 & 2 & 0.01 & 0.01 \\
\hline Grey butcherbird & Cracticus torquatus & 1 & $<0.01$ & $<0.01$ & 3 & 0.03 & 0.02 \\
\hline Australian magpie & Gymnorhina tibicen & 1 & $<0.01$ & $<0.01$ & & & \\
\hline Pied currawong & Strepera graculina & 7 & 0.05 & 0.02 & 9 & 0.09 & 0.04 \\
\hline Grey currawong & S. versicolor & 9 & 0.04 & 0.01 & 8 & 0.03 & 0.01 \\
\hline Australian raven & Corvus coronoides & 6 & 0.03 & 0.02 & 2 & 0.01 & 0.01 \\
\hline White winged chough & Corcorax melanorhamphos & & & & 1 & 0.02 & 0.02 \\
\hline Satin bowerbird & Ptilonorhynchus violaceus & 4 & 0.07 & 0.04 & & & \\
\hline Red browed finch & Neochmia temporalis & 3 & 0.01 & 0.01 & & & \\
\hline Beautiful firetail & Stagonopleura bella & 16 & 0.17 & 0.04 & 2 & 0.01 & 0.01 \\
\hline Mistletoebird & Dicaeum hirundinaceum & 8 & 0.02 & 0.01 & 9 & 0.03 & 0.01 \\
\hline Welcome swallow & Hirundo neoxena & 4 & 0.04 & 0.02 & 3 & 0.02 & 0.01 \\
\hline Tree martin & H. nigricans & 22 & 0.71 & 0.24 & 5 & 0.04 & 0.02 \\
\hline Silvereye & Zosterops lateralis & 30 & 1.58 & 0.17 & 23 & 0.19 & 0.05 \\
\hline Bassian thrush & Zoothera lunulata & 19 & 0.13 & 0.03 & 4 & 0.01 & 0.01 \\
\hline Common blackbird $^{\mathrm{a}}$ & Turdus merula & 12 & 0.05 & 0.01 & & & \\
\hline
\end{tabular}

The number of sites $(n=30)$ in riparian or non riparian habitat at which each species was recorded is also presented. a Introduced species.

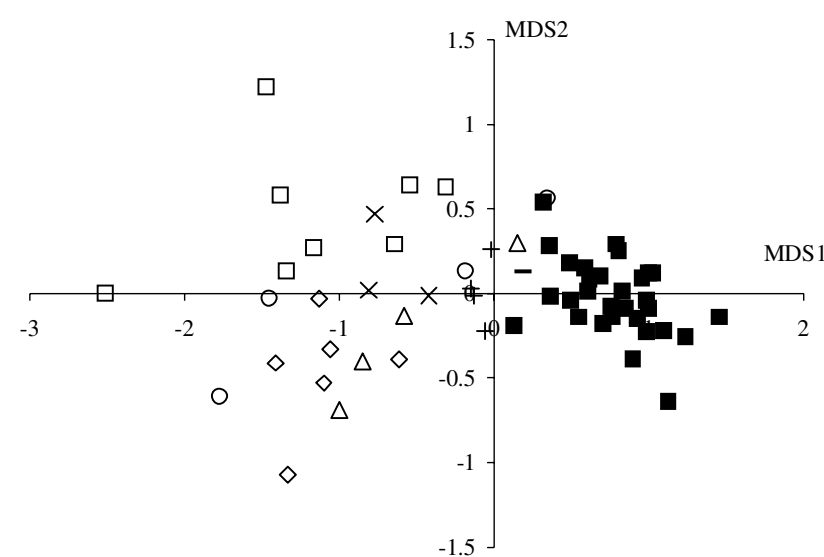

Fig. 1 - Ordination of bird assemblages occurring at sites in the Victorian Highlands (stress $=0.1$ ). The ecological vegetation class for the site at which each assemblage occurs is displayed: Riparian Forest $(\square)$, Wet Forest $(-)$, Damp Forest $(O)$, Shrubby Foothill Forest $(\Delta)$, Herb-rich Foothill Forest (+), Lowland Forest $(\diamond)$, Heathy Dry Forest $(x)$ and Heathy Woodland ( $\square)$.

non riparian habitats (Fig. 1). These included high densities of trees in the $\leqslant 10 \mathrm{~cm}$ dbh $\left(r_{\mathrm{s}} \quad 0.631, p<0.01\right), 1120 \mathrm{~cm} \mathrm{dbh}$ $\left(r_{\mathrm{s}} \quad 0.724, p<0.01\right)$ and $2140 \mathrm{~cm} \mathrm{dbh}\left(r_{\mathrm{s}} \quad 0.724, p<0.01\right)$ size classes, shrub richness $\left(r_{\mathrm{s}} \quad 0.666, p<0.01\right)$, cover of low shrubs $<1 \mathrm{~m}\left(r_{\mathrm{s}} \quad 0.606, p<0.01\right)$ and cover of grasses ( $\left.r_{\mathrm{s}} \quad 0.599, p<0.01\right)$.

The second MDS dimension (MDS2) was not as readily interpretable as MDS1. It represents a gradient from sites with a high density of trees of smaller diameter and a dense low shrub layer, to sites with larger trees, of increased height, and a dense ground fern layer (Fig. 1).

\section{Discussion}

\subsection{Landscape pattern and bird assemblages}

The value of riparian habitats for birds in mesic forests of the Victorian Highlands is disproportionately high compared with the extent of riparian vegetation in the forest landscape $(<10 \%$ of the area). The ecological value of these habitats is evi denced by the higher richness, diversity and abundance of bird species that they support, and by the distinctive compo sition of the avifauna which complements that occurring in adjacent habitats. These observations from continuous forest are consistent with the findings from studies of riparian zones in arid and semi arid environments (Shurcliff, 1980; Szaro and Jakle, 1985; Saab, 1999; Aumann, 2001), and of rem nant riparian vegetation in developed landscapes (Warkentin et al., 1995; Fisher and Goldney, 1997; Rottenborn, 1999; Miller et al., 2003), and amongst plantation and production forests 
(Friend, 1982; Armstrong and van Hensbergen, 1994; Linden mayer et al., 2002; Conner et al., 2004). The high value of ripar ian habitats for wildlife has been linked to a number of factors associated with the riparian zone, including greater availability of water (Gregory et al., 1991), increased habitat complexity (Bull and Skovlin, 1982; Douglas et al., 1992), great er levels of food resources (Gray, 1993; Murakami and Nakano, 2002), and the benefits associated with multiple edge effects (Gates and Giffen, 1991).

The influence of riparian habitats in shaping bird assem blages in mesic forest landscapes in this study is emphasised by several factors. First, riparian assemblages might have been expected to be less distinct given the relatively small distances between paired riparian and non riparian sites $(<1 \mathrm{~km})$. Sec ond, the mobility of birds, coupled with the continuity of forest habitat between riparian and non riparian sites, also contrib utes to an expectation of greater similarity between habitat types. Third, in temperate and mesic forests the more subtle gradient in vegetation structure away from streams (cf. dry environments) can be expected to have less impact on the structure of bird assemblages (McGarigal and McComb, 1992; Catterall et al., 2001). However, despite the relatively narrow width and limited extent of riparian vegetation in the forest mosaic, marked differences in the structure and composition of bird communities between riparian and non riparian sites clearly show that riparian habitats have a strong influence on the distributional patterns of birds in this forest landscape.

Five broad groups of species can be distinguished in this study area, based on their distributional patterns. Forest gener alists (36\% of all species) are species that are widespread throughout the forested landscape; riparian and non riparian sites each supported between $25 \%$ and $75 \%$ of all individuals recorded (e.g. brown thornbill, striated thornbill, spotted par dalote, grey shrike thrush, crimson rosella, grey fantail and white throated treecreeper). Overall, much of the avifauna of this study area is composed of species with widespread dis tributions throughout southeast Australia (Blakers et al., 1984; Loyn, 1985; Emison et al., 1987; Brown et al., 1989; Barrett et al., 2003) and predictably these were found throughout the landscape mosaic. Many of these species, although wide spread, were more abundant in riparian than non riparian habitats.

Riparian habitats were characterised by a suite of species more typical of wetter forest types in south east Australia. Many of these species typically had a restricted distribution in the forest mosaic. Riparian selective species (7\%) are those that occurred exclusively in riparian habitats (e.g. yellow tufted honeyeater, pink robin, satin bowerbird and the intro duced common blackbird), while riparian associated species (43\%) were strongly linked to riparian habitats (i.e. $>75 \%$ of all individuals were from riparian sites), although they also occurred in non riparian habitats, particularly wetter vegeta tion types (e.g. red browed treecreeper, large billed scrubw ren, Lewin's honeyeater, rose robin, eastern whipbird, olive whistler, rufous fantail and beautiful firetail). Several such species have core ranges centred on rainforests and closed forests of coastal central and northern Australia, and are uncommon in Victoria (e.g. large billed scrubwren and Le win's honeyeater) (Loyn et al., 1980; Emison et al., 1987; Bar rett et al., 2003).
In contrast, several species recorded at non riparian sites were conspicuously absent from, or seldom occurred in, ripar ian habitats. Notably, many of these species were most prom inent in the low, open heathy woodland communities, which were the most distinct from riparian habitats in structure, flo ristic composition and bird composition. Non riparian selective species $(2 \%)$ are those birds that occurred exclusively in non riparian habitats (e.g. buff rumped thornbill and yellow thornbill) while non riparian associated species (10\%) are those strongly linked to non riparian habitats (i.e. supporting $>75 \%$ of all individuals), although they also occurred in riparian habitats (e.g. scarlet robin, southern emu wren, rufous whis tler and olive backed oriole).

Any classification of birds in relation to riparian habitats is likely to be scale specific (Kinley and Newhouse, 1997; Woin arski et al., 2000), or responsive to other factors such as land scape position (Knopf, 1985; Finch, 1989), such that the specific composition of groups cannot necessarily be general ised between regions. For example, in the dry box ironbark forests of central Victoria, Mac Nally et al. (2000) recorded dis tributional patterns for a range of species occurring at 'gully' (intermittent stream channels) and ridge sites, including a number of species common to this study. There, the red wat tlebird and eastern rosella were among species which were more abundant in gullies and which contributed strongly to compositional differences between gully and ridge sites. In this study, both species were more abundant in non riparian habitats (Table 3). Thus, while the underlying principle is the same, that riparian zones support high bird species richness and abundance and distinct assemblages, species affinities may differ across large spatial scales.

\subsection{Habitat characteristics and bird assemblages}

Structural complexity of habitats has long been known to influence avian species richness and composition (MacArthur and MacArthur, 1961; Willson, 1974; Cody, 1981) and fre quently has been cited as a key factor to explain contrasts be tween bird assemblages of riparian zones and surrounding habitats (Hubbard, 1977; Emmerich and Vohs, 1982; Finch, 1989). In this study, riparian habitats were floristically and structurally distinct from adjacent upland vegetation and consequently their presence promotes habitat diversity across the forest landscape. Riparian habitats have a more complex vegetation structure, including a mid storey tree layer largely absent from non riparian habitats. They also support plant species and associations not generally found in non riparian situations. For example, eucalypts of the sub genus Symphyomyrtus (e.g. E. viminalis, E. camphora and E. ovata) are dominant in riparian situations, while species of the sub genus Monocalyptus (e.g. E. obliqua, E. radiata, E. sieberi and E. baxteri) tend to dominate non riparian habitats (Austin et al., 1996; Catterall et al., 2001).

While habitat structural complexity has been associated with greater richness and abundance of bird assemblages in riparian zones (Douglas et al., 1992; Sanders and Edge, 1998), less emphasis has been given to floristic composition in shaping the avifauna of riparian habitats. In this study, both physiognomic and floristic differences between habitat types influence bird assemblages. For example, the complex 
mid storey of riparian vegetation provides favoured foraging habitat for several species characteristic of riparian habitats (e.g. rose robin, Lewin's honeyeater and golden whistler). Sim ilarly, the occurrence of a number of bark foraging species (e.g crested shrike tit and white eared honeyeater) was closely associated with that of bark decorticating eucalypts (e.g. E. viminalis, E. camphora and to a lesser degree E. radiata), which predominate in riparian zones (Austin et al., 1996). Birds more typical of non riparian habitats include several that favour the more open ground layer for foraging, including buff rum ped thornbill and scarlet robin. Indeed, consideration of com munity level measures (e.g. richness, diversity) in isolation may mask the interrelated influences of physiognomic and floristic factors on bird communities. The taxonomic diversity and the wide range of ecological requirements among species strongly associated with riparian zones (i.e. riparian selective and riparian associated species), suggests that the riparian influence is unlikely to be due to a specific structural feature or floristic characteristic (Woinarski et al., 2000).

\subsection{Implications for conservation}

Riparian habitats are important for avifaunal conservation in continuous forest landscapes for at least five reasons. First, the vegetation differs in both floristic composition and struc tural complexity from that of adjacent non riparian habitats. Thus, riparian zones add to the diversity of the landscape mo saic and to the diversity of habitats and resources available to forest birds. Second, a suite of bird species are strongly asso ciated with, or predominantly confined to, the riparian zone. These species are likely to occur in relatively lower abun dance (or be absent) from the forest landscape if not for the presence of riparian vegetation. Third, most forest bird spe cies use riparian habitats at some stage of their life, and more than a third of all species (36\%) attained higher densities in riparian habitats than in other forest types. Fourth, the dis tinctiveness of riparian vegetation and the prevalence of bird species typical of wet forests, suggest that they may function as seasonal or refuge habitats when conditions become stressful in upland habitats. This includes the potential for these habitats to function as drought and fire refuges (Nix, 1993). Last, riparian habitats in this study area are known to be used by several species of threatened conservation status, including the powerful owl Ninox strenua and sooty owl Tyto tenebricosa (Loyn et al., 2001).

While riparian habitats characteristically support richer and more abundant assemblages, they comprise only a small proportion of the forest landscape ( $<10 \%$ of the total area). Most of the landscape consists of non riparian forest and it is these forests, by virtue of their greater area, that serve as the major population reservoirs for most species of forest birds. Consequently, the ecological role and value of non riparian habitats should not be overlooked. Further, riparian habitats are not suitable for all species (McGarigal and McComb, 1992; Murray and Stauffer, 1995; Mac Nally et al., 2000). In this study, a number of species clearly were associ ated with non riparian habitats, including at least $12 \%$ of spe cies classed as non riparian selective and non riparian associated species. Clearly, the maintenance of diverse and sustainable assemblages of birds in forest landscapes depends on complementary management of both riparian and non riparian vegetation types. This highlights the importance of landscape level planning and management for avifaunal con servation in forest mosaics.

\section{Acknowledgements}

A Deakin University Postgraduate Research Scholarship (to G.P.) greatly assisted this research. Grants from the Holsworth Wildlife Research Endowment and the Stuart Leslie Bird Re search Award (Birds Australia) provided further assistance with field costs, and are gratefully acknowledged. Research was conducted pursuant to the provisions of the Victorian $\mathrm{Na}$ tional Parks Act 1975, under Permit Number 10001565. We thank two anonymous referees for their careful comments on the manuscript.

\section{R E F E R E N C E S}

Anderson, B.W., Ohmart R.D., 1977. Vegetation structure and bird use in the Lower Colorado River Valley. In: Johnson, R.R., Jones, D.A. (Eds.), Importance, Preservation and Management of Riparian Habitat: A Symposium. United States Department of Agriculture Forest Service, Tucson, Arizona, pp. 2334.

Armstrong, A.J., van Hensbergen, H.J., 1994. Comparison of avifaunas in Pinus radiata habitats and indigenous riparian habitat at Jonkershoek, Stellenbosch. South African Journal of Wildlife Research 24, 4855

Aumann, T., 2001. The structure of raptor assemblages in riparian environments in the south west of the Northern Territory, Australia. Emu 101, 293304.

Austin, M.P., Pausas, J.G., Nicholls, A.O., 1996. Patterns of tree species richness in relation to environment in southeastern New South Wales, Australia. Australian Journal of Ecology 21, 154164.

Barrett, G., Silcocks, A., Barry, S., Cunningham, R.B., Poulter, R., 2003. The New Atlas of Australian Birds. Royal Australasian Ornithologists Union, Melbourne

Blakers, M., Davies, S.J.J.F., Reilly, P.N., 1984. The Atlas of Australian Birds. Melbourne University Press, Melbourne.

Borchert, M., 2003. Environmental relationships of riparian birds in the transverse ranges of southern California. In: Faber, P.M. (Ed.), 2001 Riparian Habitat and Flood plains Conference Proceedings. Riparian Habitat Joint Venture, Sacremento, pp. 212.

Brown, G.W., Earl, G.E., Griffiths, R.C., Horrocks, G.F.B., Williams, L.M., 1989. Flora and Fauna of the Acheron Forest Block, Central Highlands, Victoria. Ecological Survey Report No. 30. Department of Conservation, Forests and Lands, Melbourne.

Bull, E.L., Skovlin, J.M., 1982. Relationships between avifauna and streamside vegetation. Transactions of the North American Wildlife Natural Resources Conference 47, 496506.

Catterall, C.P., 1993. The importance of riparian zones to terrestrial wildlife. In: Bunn, S.E., Pusey, B.J., Price, P. (Eds.), Ecology and Management of Riparian Zones in Australia, Land and Water Resources Research and Development Corporation, Canberra, pp. 4152.

Catterall, C.P., Piper, S.D., Bunn, S.E., Arthur, J.M., 2001. Flora and fauna vary with local topography in a subtropical eucalypt forest. Austral Ecology 26, 5669.

Christidis, L., Boles, W.E., 1994. The Taxonomy and Species of Birds of Australia and its Territories. Royal Australasian Ornithologists Union, Hawthorn East. 
Clarke, K.R., Gorley, R.N., 2001. PRIMER v5: User Manual/Tutorial. Plymouth Marine Laboratory, PRIMER E Ltd, Plymouth.

Cody, M.L., 1981. Habitat selection in birds: the roles of vegetation structure, competitors and productivity. BioScience 31, 107113.

Cody, M.L., 1993. Bird diversity components within and between habitats in Australia. In: Ricklefs, R.E., Schluter, D. (Eds.), Species Diversity in Ecological Communities: Historical and Geographical Perspectives. The University of Chicago Press, Chicago, pp. 147158.

Commonwealth and Victorian Regional Forest Agreement Steering Committee, 1997. Central Highlands Comprehensive Regional Assessment Report. Commonwealth and Victorian Regional Forest Agreement Steering Committee, Canberra.

Conner, R.N., Dickson, J.G., Williamson, J.H., Ortego, B., 2004. Width of streamside zones and breeding bird abundance in eastern Texas. Southeastern Naturalist 3, 669682.

Crome, F., Isaacs, J., Moore, L., 1995. The utility to birds and mammals of remnant riparian vegetation and associated windbreaks in the tropical Queensland uplands. Pacific Conservation Biology 1, 328343.

Decamps, H., Joachim, J., Lauga, J., 1987. The importance for birds of the riparian woodlands within the alluvial corridor of the River Garonne, S.W. France. Regulated Rivers 1, 301316.

Douglas, D.C., Ratti, J.T., Alan, B., Alldredge, J.R., 1992. Avian habitat associations in riparian zones of Idaho's Centennial Mountains. Wilson Bulletin 104, 485500.

Doyle, A.T., 1990. Use of riparian and upland habitats by small mammals. Journal of Mammalogy 71, 1423.

Emison, W.B., Beardsell, C.M., Norman, F.I., Loyn, R.H., 1987. Atlas of Victorian Birds. Department of Conservation, Forests and Lands and Royal Australian Ornithologists Union, Melbourne.

Emmerich, J.M., Vohs, P.A., 1982. Comparative use of four woodland habitats by birds. Journal of Wildlife Management 46, 4349.

Finch, D.M., 1989. Habitat use and habitat overlap of riparian birds in three elevational zones. Ecology 70, 866880.

Fisher, A.M., Goldney, D.C., 1997. Use by birds of riparian vegetation in an extensively fragmented landscape. Pacific Conservation Biology 3, 275288.

Fleishman, E., Murphy, D.D., Floyd, T., McDonal, N., Walters, J., 2002. Characterization of riparian bird communities in a Mojave Desert watershed. Great Basin Birds 5, 3844.

Forman, R.T.T., 1995. Land Mosaics: The Ecology of Landscapes and Regions. Cambridge University Press, Cambridge.

Friend, G.R., 1982. Bird populations in exotic pine plantations and indigenous eucalypt forests in Gippsland, Victoria. Emu 82, 8091.

Gates, J.E., Giffen, N.R., 1991. Neotropical migrant birds and edge effects at a forest stream ecotone. Wilson Bulletin 103, 204217.

Gray, L.J., 1993. Response of insectivorous birds to emerging aquatic insects in riparian habitats of a tallgrass prairie stream. American Midland Naturalist 129, 288300.

Gregory, S.V., Swanson, F.J., McKee, W.A., Cummins, K.W., 1991. An ecosystem perspective of riparian zones. Bioscience 41, 540551.

Hodges, M.F.J., Krementz, D.G., 1996. Neotropical migratory breeding bird communities in riparian forests of different width along Altamaha River, Georgia. Wilson Bulletin 108, 496506.

Hubbard, J.P. 1977. Importance of riparian ecosystems: biotic considerations. In: Johnson, R.R., Jones, D.A. (Eds.), Importance, Preservation and Management of Riparian Hab itat: A Symposium. United States Department of Agriculture Forest Service, Tucson, Arizona, pp. 1418.

Jansen, A., Robertson, A.I., 2001. Riparian bird communities in relation to land management practices in floodplain woodlands of southeastern Australia. Biological Conservation 100, 173185.

Kilgo, J.C., Sargent, R.A., Chapman, B.R., Miller, K.V., 1998. Effect of stand width and adjacent habitat on breeding bird communities in bottomland hardwoods. Journal of Wildlife Management 62, 7283.

Kinley, T.A., Newhouse, N.J., 1997. Relationship of riparian reserve zone width to bird density and diversity in southeastern British Columbia. Northwest Science 71, 7586.

Knopf, F.L., 1985. Significance of riparian vegetation to breeding birds across an altitudinal cline. In: Johnson, R.R., Ziebell, C.D., Paton, D.C., Ffolliott, P.F., Homre, R.H. (Eds.), Riparian Ecosystems and their Management: Reconciling Conflicting Uses. First North American Riparian Conference. US Forest Service, Fort Collins, pp. 105111.

Knopf, F.L., Johnson, R.R., Rich, T., Samson, F.B., Szaro, R., 1988. Conservation of riparian ecosystems in the United States. Wilson Bulletin 100, 272284.

Knopf, F.L., Samson, F.B., 1994. Scale perspectives on avian diversity in western riparian ecosystems. Conservation Biology 8, 669676.

Lachavanne, J. B., 1997. Why study biodiversity in land inland water ecotones. In: Lachavanne, J. B., Juge, R. (Eds.), Biodiversity in Land Inland Water Ecotones. UNESCO and The Parthenon Publishing Group, Paris, pp. 145.

Lindenmayer, D.B., Cunningham, R.B., Donnelly, C.F., Nix, H., Lindenmayer, B.D., 2002. Effects of forest fragmentation on bird assemblages in a novel landscape context. Ecological Monographs 72, 118.

Loyn, R.H., 1985. Ecology, distribution and density of birds in Victorian forests. In: Keast, A., Recher, H.F., Ford, H., Saunders, D. (Eds.), Birds of Eucalypt Forests and Woodlands, Ecology, Conservation and Management. Surrey Beatty \& Sons, Chipping Norton, pp. 3346.

Loyn, R.H., MacFarlane, M.A., Chesterfield, E.A., Harris, J.A., 1980. Forest Utilisation and the Flora and Fauna of Boola Boola State Forest in South eastern Victoria. Bulletin No. 28. Forest Commission Victoria, Melbourne.

Loyn, R.H., McNabb, E.G., Volodina, L., Willig, R., 2001. Modelling landscape distributions of large forest owls as applied to managing forests in north east Victoria, Australia. Biological Conservation 97, 361376.

Lynch, R.J., Catterall, C.P., 1999. Riparian wildlife and habitats. In: Lovett, S.E., Price, P. (Eds.). Riparian Land Management Technical Guidelines. Land and Water Resources Research and Development Corporation, Canberra, pp. A121 A136.

Mac Nally, R., Soderquist, T., Tzaros, C.L., 2000. The conservation value of mesic gullies in dry woodland landscapes: avian communities in the box ironbark system of southern Australia. Biological Conservation 93, 293302.

MacArthur, R.H., MacArthur, J.W., 1961. On bird species diversity. Ecology 42, 594598.

Malanson, G.P., 1993. Riparian Landscapes. Cambridge University Press, Cambridge.

Martin, T.G., McIntyre, S., Catterall, C.P., Possingham, H.P., 2006. Is landscape context important for riparian conservation? Birds in grassy woodland. Biological Conservation 127, 201214.

McGarigal, K., McComb, W.C., 1992. Streamside versus upslope breeding bird communities in the central Oregon Coast Range. Journal of Wildlife Management 56, 1023.

Miller, J.R., Wiens, J.A., Hobbs, N.T., Theobald, D.M., 2003. Effects of human settlement on bird communities in lowland riparian areas of Colorado (USA). Ecological Applications 13, 10411059.

Mueller Dombois, D., Ellenberg, H., 1974. Aims and Methods of Vegetation Ecology. John Wiley \& Sons, New York.

Murakami, M., Nakano, S., 2002. Indirect effect of insect emergence on a terrestrial insect population through by birds predation. Ecology Letters 5, 333337. 
Murray, N.L., Stauffer, D.F., 1995. Nongame bird use of habitat in central Appalachian riparian forests. Journal of Wildlife Management 59, 7888.

Nix, H.A., 1993. Bird distributions in relation to imperatives for habitat conservation in Queensland. In: Catterall, C.P., Driscoll, P., Hulsman, K.A., Taplin, A. (Eds.), Birds and their Habitats: Status and Conservation in Queensland. Queensland Ornithological Society, Brisbane, pp. 1221.

Pearson, S.F., Manuwal, D.A., 2001. Breeding bird response to riparian buffer width in managed Pacific Northwest Douglas Fir forests. Ecological Applications 11, 840853.

Pyke, G.H., Recher, H.F., 1984. Censusing Australian birds: a summary of procedures and a scheme for standardisation of data presentation and storage. In: Davies, S.J.J.F. (Ed.), Methods of Censusing Birds in Australia. Department of Conservation and Environment, Perth, pp. 5563.

Robertson, A., Jarvis, A.M., Brown, C.J., 1998. Avian diversity and endemism in Namibia: patterns from the Southern African Atlas Project. Biodiversity and Conservation 7, 495511.

Rottenborn, S.C., 1999. Predicting the impacts of urbanization on riparian bird communities. Biological Conservation 88, 289299.

Saab, V., 1999. Importance of spatial scale to habitat use by breeding birds in riparian forests: a hierarchical analysis. Ecological Applications 9, 135151.

Sanders, T.A., Edge, W.D., 1998. Breeding bird community composition in relation to riparian vegetation structure in the western United States. Journal of Wildlife Management 62, 461473.
Shirley, S.M., Smith, J.N.M., 2005. Bird community structure across riparian buffer strips of varying width in a coastal temperate forest. Biological Conservation 125, 474489.

Shurcliff, K.S., 1980. Vegetation and bird community characteristics in an Australian arid mountain range. Journal of Arid Environments 3, 331348.

Stauffer, D.F., Best, L.B., 1980. Habitat selection by birds of riparian communities: evaluating effects of habitat alterations. Journal of Wildlife Management 41, 115

Szaro, R.C., 1980. Factors influencing bird populations in southwestern riparian forests. In: DeGraff, R.M. (Ed.), Management of Western Forests and Grasslands for Nongame Birds. US Forestry Service, pp. 403418.

Szaro, R.C., Jakle, M.D., 1985. Avian use of a desert riparian island and its adjacent scrub habitat. Condor 87, 511519.

Thomas, J.W., Maser, C., Rodiek, J.E., 1978. Riparian zones in managed rangelands their importance to wildlife. In: Thomas, J.W. (Ed.), Grazing and Riparian/stream Ecosystems. Trout Unlimited, Denver, Colorado, pp. 2131.

Warkentin, I.G., Greenberg, R., Salgado Ortiz, J., 1995. Songbird use of gallery woodlands in recently cleared and older settled landscapes of the Selva Lacandona, Chiapas, Mexico. Conservation Biology 9, 10951106.

Willson, M.F., 1974. Avian community organization and habitat structure. Ecology 55, 10171029.

Woinarski, J.C.Z., Brock, C., Armstrong, M., Hempel, C., Cheal, D., Brennan, K., 2000. Bird distribution in riparian vegetation in the extensive natural landscape of Australia's tropical savanna: a broad scale survey and analysis of a distributional data base. Journal of Biogeography 27, 843868. 